\title{
Croissance urbaine et insécurité dans la ville de Niamey
}

\author{
Kokou Henri Motcho, Niamey
}

\section{Introduction}

La rapidité et l'ampleur de la croissance démographique et spatiale de Niamey n'ont pas permis aux pouvoirs publics de doter la capitale du Niger de l'ensemble des infrastructures collectives nécessaires à la vie quotidienne des citadins. Cette crise de la gestion urbaine se traduit non seulement par une détérioration du cadre de vie des habitants mais aussi par une insécurité grandissante qui est malheureusement le résultat de deux facteurs principaux intimement liés: la paupérisation grandissante de la population et la faiblesse du mode d'encadrement des jeunes. La délinquance juvénile prend de plus en plus d'ampleur comme en témoigne le très grand nombre d'enfants de la rue, qui, à l'occasion, commettent de petits larcins ou sont les indicateurs des grands délinquants.

Quelle signification faut-il donner à ce nouveau phénomène de société? Les statistiques permettent-elles de vérifier une augmentation de la criminalité d'année en année, comme le laissent entendre les services de la police et la presse à sensation qui s'intéresse depuis peu à la criminalité? Enfin la croissance urbaine rimet-elle avec une augmentation de la criminalité? Autant de questions auxquelles nous tenterons d'apporter une réponse.

\section{Urbanisation et insécurité}

La relation entre la démographie galopante des villes et les problèmes sociaux que connaissent les villes africaines est controversée. En effet, beaucoup de spécialistes des villes du tiers-monde posent la question de savoir si la rapidité de la croissance démographique n'engendre pas des problèmes sociaux au nombre desquels la délinquance figure en bonne place, compte tenu de la concentration de la population sur un espace réduit.

Les variables démographiques (évolution et structure de la population, densités par quartiers) sont, en effet, des facteurs non négligeables dans le développement de l'insécurité. Curieusement, elles ne sont pas toujours prises en compte. Or, comment peut-on raisonnablement penser l'avenir sans tenir compte des projections démographiques qui permettent d'anticiper des changements sociaux et politiques de grande ampleur?
La rapidité de la croissance démographique comporte un certain nombre de conséquences perceptibles dans les structures démographiques et les densités par quartier, dont nous essaierons de rendre compte pour expliquer les facteurs favorisant la délinquance.

\subsection{Croissance urbaine et sentiment d'insécurité}

Petit village dans les années trente, Niamey est devenu, en 1952, pour la première fois, le premier centre urbain du Niger, avec 11790 habitants; en 1972, sa population dépassa les 100000 habitants. A partir de cette date, l'explosion démographique a été plus rapide encore que durant la période précédente. Aujourd'hui la ville compte près de 700000 habitants dont un grand nombre d'actifs est frappé par le chômage, le sousemploi et le travail temporaire, contribuant ainsi à l'hypertrophie du travail informel. La détérioration de l'emploi formel ne peut être dissociée du phénomène de la délinquance urbaine.

L'exceptionnelle croissance de la population s'est traduite par une extension rapide de la ville (Fig. 1): en 1970, Niamey couvrait 1367 ha alors qu'aujourd'hui elle s'étend sur 10384 ha et sur les deux rives du fleuve Niger. Elle ne compte, cependant, que cinq commissariats de police de proximité coiffés par un Commissariat Central $(\mathrm{CCN})$ et une Direction de la Police Judiciaire (DPJ), à compétence nationale. La répartition de ces commissariats est très inégale: la rive droite du fleuve Niger et la rive ouest du Gounti Yéna, qui divise en deux le plateau de la rive gauche, ne comptent chacun qu'un seul commissariat contre 3 pour la rive Est du talweg qui abrite aussi le CCN et la DPJ.

L'insuffisance de commissariats de police se traduit par une insécurité grandissante: vols à la tire, cambriolages et agressions, contre lesquels les fonctionnaires de la police sont impuissants.

Niamey, à l'instar des autres villes d'Afrique noire, a un paysage hétérogène. Composée de trois communes, on y dénombre quatre types de tissus urbains :

\subsubsection{Les quartiers modernes}

Ils sont bâtis selon les normes résidentielles de la métropole: villas entourées de pelouses occupant le plus souvent de grandes parcelles de 600 à $1200 \mathrm{~m}^{2}$, et disposant d'un équipement sanitaire complet. Ces quartiers sont peu peuplés et souvent situés soit en zone péri-centrale (le quartier Plateau et ses extensions) soit à la périphérie (Kouara Kano). Faible densité de population, situation géographique et aisance des habitants sont autant de facteurs attirant les 


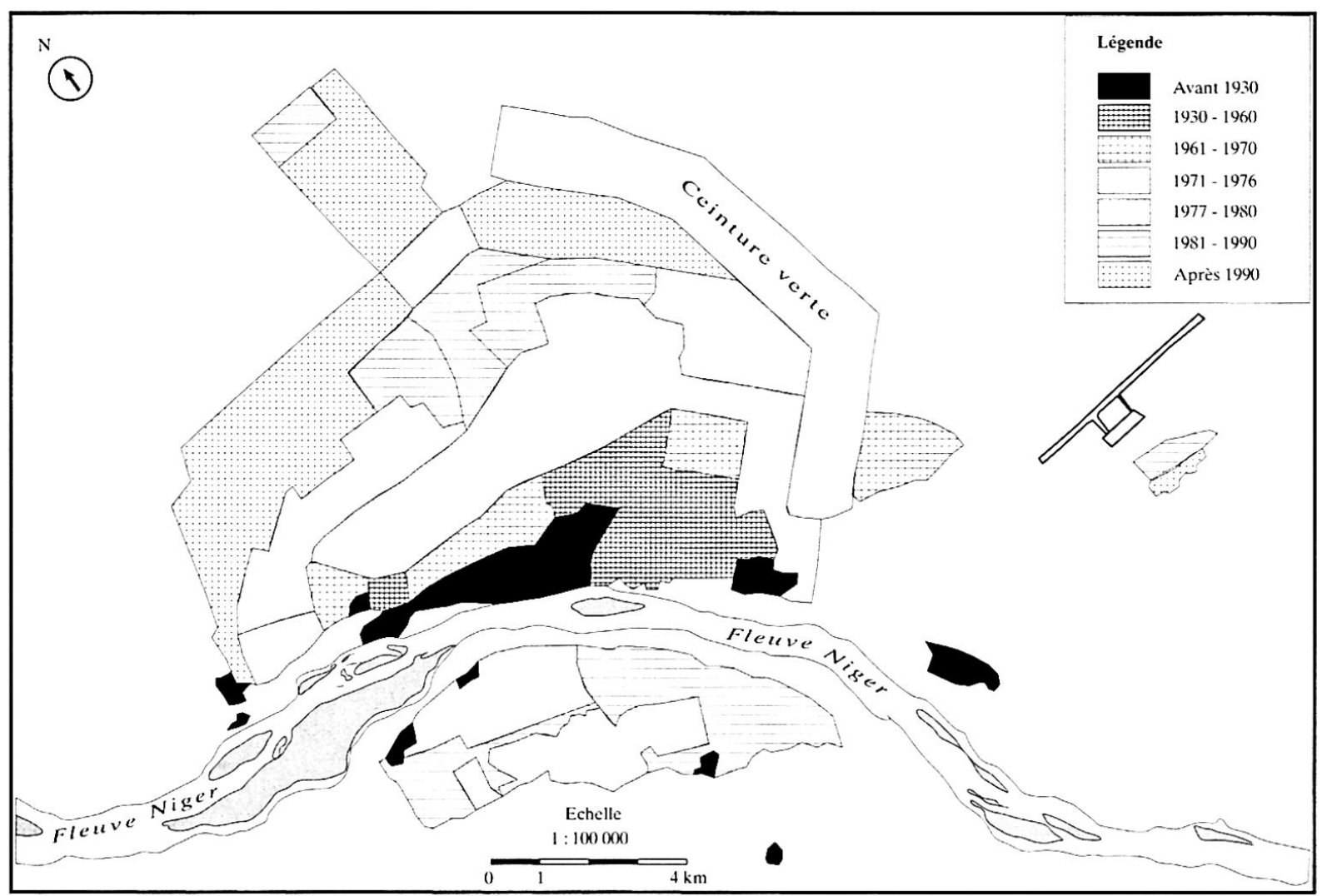

Fig. 1: Quelques étapes de la croissance spatiale de Niamey Different stages of spatial development in Niamey

Phasen des räumlichen Wachstums von Niamey

Source: H.K. Motcho; dessin: D. LAWALI

cambrioleurs. Bijoux, antennes et postes de télévision, appareil hi-fi, véhicules sont les articles les plus prisés.

Aussi, pour lutter contre ces délinquants, les habitants de ces quartiers transforment de plus en plus leurs maisons en bunker par la fixation de grilles de protection aux fenêtres, et de tôle sur les grilles d'entrée des villas. Toutes ces précautions prises pour prévenir les agressions extérieures le sont malheureusement au détriment des mesures de sécurité contre les incendies internes. On sacrifie aussi la beauté de l'architecture à la sécurité, en entourant les villas autrefois clôturées par un simple grillage ou des murets surmontés de claustras ou grilles dont la hauteur atteint à peine deux mètres, par des murs hauts de quatre à cinq mètres surmontés de pointes ou de tessons de bouteilles. En plus de ce dispositif de sécurité, on plante également des bougainvilliers qui recouvrent le mur de clôture et dont les innombrables épines dissuadent les visiteurs indésirables.

Ainsi, dans les quartiers modernes, le réflexe sécuri- taire se traduit d'une part aujourd'hui par une dissimulation des belles architectures invisibles de l'extérieur et aussi de l'intérieur à cause de l'absence de perspective; et d'autre part par l'utilisation des services des sociétés de gardiennage pour les plus nantis, de gardiens de nuits pour les autres et enfin l'acceptation volontaire de contribuer aux petites dépenses des milices privées qui font des rondes dans le quartier. Ces milices appelées "Yam banga» constituées de jeunes souvent sans emploi sont rétribuées par des cotisations volontaires fixées à $500 \mathrm{~F}$ CFA par ménage. Mal encadrées, ces milices exécutent les voleurs pris la main dans le sac, au grand dam de la Police et de la Justice mais à la satisfaction des populations.

\subsubsection{La ville traditionnelle}

On y distingue trois types de quartiers en fonction de leur situation par rapport au centre ville et à la criminalité:

\section{La cité intérieure}

C'est un tout autre type de paysage; la cité intérieure correspond à l'ancienne «ville indigène» et est, de 
ce fait, constituée de quartiers populaires situés aujourd'hui dans le centre-ville. Cette cité est caractérisée par:

- la forte densité de population (772,7 habitants par hectare);

- le vieillissement et l'obsolescence de l'habitat qui est de type cour en banco;

- la concentration des établissements éducatifs, sanitaires et commerciaux;

- la compétition pour le sol à travers l'expansion des fonctions commerciales qui s'organisent autour du Petit-Marché, du Grand-Marché et du marché Katako. Boutiques et étalages se succèdent le long des rues qui mènent à ces marchés. Ailleurs aussi le micro-commerce est omniprésent.

L'activité commerciale, qui est de loin la plus importante dans la cité, attire tous les laissés-pour-compte de la ville. Vols à la tire, à l'étalage et cambriolage des boutiques y sont par conséquent très fréquents, malgré la vindicte populaire (lynchage).

\section{Les quartiers traditionnels péri-urbains}

Le style et la densité de population sont identiques à ceux des quartiers de la cité intérieure mais le niveau d'équipement est plus faible: les rues sont en terre et non éclairées. L'activité économique se résume ici au micro-commerce présent dans les rues. Ces quartiers sont confrontés aux mêmes problèmes de criminalité: bagarres, vols et cambriolages des boutiques et des habitations. Dans ces quartiers, les effractions ont lieu pendant la saison sèche et chaude lorsque les habitants dorment la nuit à la devanture de leur logement; les cambrioleurs percent le mur de banco après l'avoir mouillé. En ce qui concerne les boutiques, ils y pénètrent après avoir démonté la toiture en tôle.

\section{Les quartiers périphériques mixtes}

Ils n'ont rien de commun avec les quartiers précédents hormis le plan qui est ici aussi en damier, mais les parcelles sont plus petites. L'habitat essentiellement en dur est occupé surtout par des ménages de cadres moyens, d'ouvriers qualifiés ou des commerçants moyens, qui habitent soit des villas soit des maisons multi-familiales. Si les villas abritent uniquement un ménage de six à sept personnes, l'habitat en cour collective abrite en moyenne trois ménages de six à sept personnes vivant dans une certaine promiscuité. Malgré le nombre élevé de personnes dans l'habitat de type cour, la densité de population dans ces quartiers demeure encore faible (225 habitants/ha) à cause de la mise en valeur insuffisante des parcelles par leurs attributaires: environ un tiers des parcelles est vide. Est-il donc étonnant que ces quartiers mal éclairés, mal desservis par le réseau routier et ne disposant pas de police de proximité soient les zones les plus criminogènes de la capitale? L'abondance de biens matériels et un tissu urbain lâche sont autant d'opportunités pour organiser des vols qualifiés et des agressions.

\subsubsection{Les villages annexés par la ville}

Ce sont les villages traditionnels aujourd'hui inclus dans le tissu urbain. Ils ont gardé une physionomie rurale, avec des ruelles serpentant entre des concessions en banco bâties dans le plus complet désordre et s'ouvrant parfois par un vestibule. La vie rurale reste encore vivace dans ces quartiers densément peuplés (600 hab./ha). Ces quartiers peuvent être scindés en deux: quartiers restructurés et non restructurés. Les premiers se trouvant en position péri-centrale sont densément peuplés. A ce titre ils enregistrent les même types de crimes que les quartiers péri-centraux. Quant aux seconds, ils sont situés à la périphérie de la ville. Ici trois types de lotissement coexistent le plus souvent: le village originel, les nouveaux lotissements et les zones d'habitat spontané. Si les villages semblent épargnés par la criminalité, les nouveaux lotissements sont soumis à des agressions continues, de sorte que c'est ici qu'on enregistre le plus grand nombre de Yam banga et pour cause, l'habitat informel abrite le plus souvent les délinquants, les débits clandestins de boisson et les prostituées qu'ils fréquentent: c'est le cas de Nialga sur la rive droite du fleuve Niger, de Rouba dans le talweg du Gounti Yéna qui divise en deux le plateau de la rive gauche ou encore de la ceinture verte qui compte plusieurs campements de paillotes où les délinquants peuvent se réfugier une fois leurs forfaits accomplis. Ces fiefs de délinquants abritent des bandes de voleurs organisés opérant dans des quartiers bien ciblés de Niamey. Chaque fief est coiffé par un chef de voleurs connu par les services de police et même des Niaméens. Il n'est pas rare que des victimes de vols de motos paient les services de ces chefs de voleurs pour récupérer leurs biens.

\subsubsection{Les quartiers spontanés non rattachés à un village}

Ce sont tous les quartiers périphériques non lotis par la Mairie de Niamey. On distingue ceux qui sont en banco comme Koubia (Commune I) et Pays Bas (Commune II), ainsi que ceux en paillote (Foulan Kouara, Kouara Tégui) qui abritent les petites gens. Coupés le plus souvent de la ville, il est hasardeux de s'y rendre à pied la nuit.

\subsection{Les contraintes démographiques: structure par âge et par sexe}

L'analyse des différentes structures démographiques de la ville de Niamey montre l'extraordinaire jeunesse de sa population. La répartition par âge de la population de Niamey nous permet de noter quelques traits intéressants de 1959 à 1988: d'une part les effectifs des moins de 20 ans ont évolué, passant de $47 \%$ à $57 \%$ et d'autre part la grande jeunesse de la population dont 
l'âge moyen est passé de 23 à 20 ans (Мотсно 1991) Cette jeunesse de la population est ambivalente dans ses conséquences, car le dynamisme potentiel qu'elle représente est freiné par les charges en matière d'éducation, de santé qu'elle entraîne, et que supportent les adultes. Cette situation suppose que les adultes, dont la part relative dans la population totale ne cesse de chuter (elle est passée de 49 à 39\% entre 1959 et 1988), doivent consacrer une part importante de leurs revenus à la formation des jeunes. Il en est de même de l'Etat qui malheureusement éprouve de plus en plus de difficultés à mobiliser les ressources garantissant à l'ensemble de la population une éducation et une formation correspondant aux exigences de l'économie moderne, assurant le développement d'un système de santé adéquat, des logements en quantité et en qualité suffisantes ou un minimum de sécurité sociale. Or, $42 \%$ des habitants de Niamey sont pauvres, selon le Ministère des Finances et du Plan (1993). Ces pauvres ont généralement des enfants de santé précaire, qui sont mal armés pour intégrer et poursuivre une scolarité primaire et qui restent ensuite le plus souvent à l'écart du marché du travail (Мотсно 1991; Motcho \& BEN ADJI 1998).

Signalons à titre d'exemple que le taux de scolarisation à Niamey est de $80 \%$, contre $36 \%$ pour l'ensemble du Niger et seulement $29 \%$ pour la zone rurale qui déverse son «trop plein de population» sur Niamey. Ce faible taux de scolarisation explique le rôle important joué par l'école coranique qui comptait en 199521600 élèves (Souley 1995) mal préparés pour la vie professionnelle et dont l'insertion urbaine pose problème. Une partie de ces élèves provenant de la campagne à la suite des pérégrinations de leur maître doivent mendier pour survivre. Formés ainsi à l'école de la rue, beaucoup versent dans la petite délinquance.

Faible taux de scolarisation, forte déperdition scolaire et non compétitivité de l'école coranique sont autant de contraintes au développement de l'économie formelle. Il en résulte une hypertrophie de l'économie informelle caractérisée, selon E. GRÉGOIRE (1992), par des relations de dépendance entre un maître et son serviteur.

"Ce dernier se voue au service de son patron et lui rend de multiples services sans attendre de rémunération fixée au préalable. En échange, le maître se doit de le gratifier de dons, parfois de commissions et prend en charge sa vie matérielle et sociale».

La promotion des jeunes dans ce secteur est de ce fait très difficile. L'exploitation économique des groupes les plus défavorisés par la minorité dominante ne va-t-elle pas entraîner une montée de la délinquance, notamment la criminalité dirigée contre les biens?

\subsection{Les contraintes économiques: vers le cercle vicieux de la pauvreté}

Plusieurs travaux consacrés à l'urbanisation de l'Afrique subsaharienne montrent l'influence néfaste de l'urbanisation rapide du continent. En un demi-siècle, Niamey a vu sa population passer de 11000 à plus de 700000 habitants. Dans l'agglomération, une croissance annuelle de 5\% représente 35000 à 50000 citadins de plus; ce sont les migrants arrivés de la campagne $(2 \%)$ et des enfants nés des familles citadines; ceux-ci comme les nouveaux venus ont à résoudre des problèmes d'emplois. Or le Niger, actuellement, confronté à des difficultés financières et pris dans l'étau de l'ajustement structurel, a réduit sévèrement ses investissements urbains depuis 1985, bloquant de ce fait les emplois urbains.

Le ralentissement de l'industrialisation dû à la fois à une fraude massive, à la saturation d'un marché étroit et à la crise économique générale, à la fermeture ou la privatisation de maintes entreprises nationales jusque-là soutenues par des fonds publics, de même que le retrait d'entreprises privées ont gonflé le rang des citadins sans salaire. Pour conjurer le problème de l'emploi,l'économie informelle s'est considérablement développée: elle comptait 20614 établissements dont $58 \%$ non sédentaires, selon une étude réalisée par le Ministère du PLAN en 1989. Cette étude corrobore la thèse de E. GrÉGoIre (1992) selon laquelle ce secteur ne permet pas la promotion des jeunes, précisant que «l'emploi salarié est rare, le versement d'un salaire régulier, indépendamment des résultats de l'entreprise, n'étant pas dans les habitudes des entrepreneurs informels. Presque tous les employés sont apprentis ou aides familiaux».

Les questions sociales que nous venons de décrire, notamment celles touchant à la population, à l'emploi et, donc à la pauvreté, vont tôt ou tard influencer la sécurité de la Communauté Urbaine de Niamey, dès lors que les pauvres continuent à être tenus à l'écart du processus de production et d'intégration sociale. Ces pauvres, grâce à la démocratie et à l'instigation de certaines élites, ont développé de nouvelles formes de sociabilité: appartenance régionale (ressortissant de tel ou tel arrondissement), ethnique (parti politique qualifié par les médias et les chercheurs de l'Est, du Nord ou de l'Ouest) ou religieux (le mouvement izala par exemple). Ces nouvelles formes de sociabilité sont pour beaucoup un tremplin pour pénétrer le système politico-administratif. Elles constituent un danger pour la sécurité publique car elles exacerbent l'intolérance ethnique et religieuse.

On ne peut manquer d'établir une relation entre, d'une part, les processus de paupérisation et d'exclusion, très visibles à Niamey et, d'autre part, les phénomènes de marginalité sociale: criminalité, drogues, prostitution et violence. 


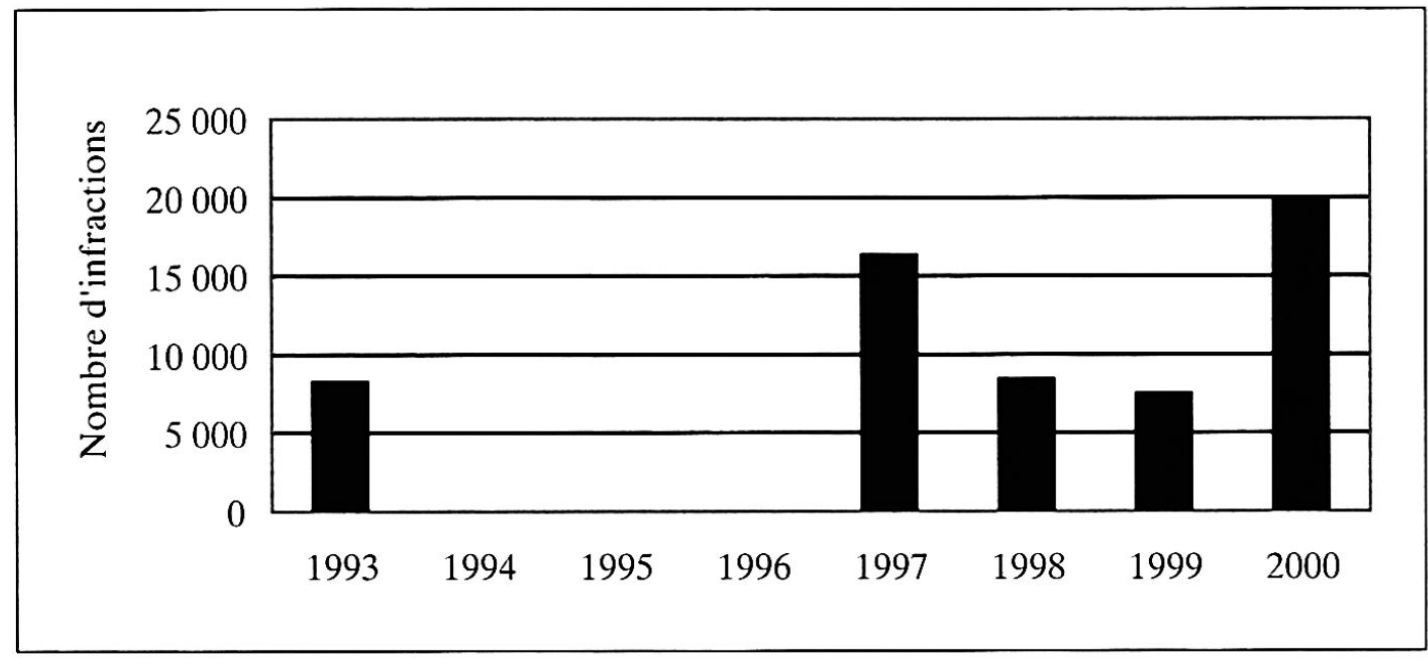

Fig. 2: Evolution des infractions enregistrées par le Service central de l'identité judiciaire (SCIJ) de 1993 à 2000 Development of transgressions registered by the Service central de l'identité judiciaire (SCIJ) between 1993-2000 Durch die Zentrale Justizbehörde registrierte Übertretungen von 1993 bis 2000

Source: Direction générale de la police nationale, Service central de l'identité judiciaire, rapports d'activités, 1993 à 2000

\section{Analyse des infractions à la loi à Niamey}

Nous allons maintenant analyser les statistiques fournies par le Service central de l'identité judiciaire (SCIJ) et le Tribunal de première instance de Niamey (TPIN), afin de vérifier si la croissance urbaine est responsable de l'augmentation des infractions à la loi.

\subsection{Le mouvement des infractions à la loi}

Les statistiques du SCIJ doivent être interprétées avec prudence car elles sont non seulement incomplètes (sur 8 ans, seules les données sur 5 années sont disponibles), mais truffées d'erreurs. Les données issues du Tribunal de première instance de Niamey (TPIN) se rapportent uniquement aux délits de vols qui, après les rafles régulièrement opérées par les services de la police, constituent les premières causes d'interpellations.

Selon les données du SCIJ, 1997 et 2000 ont été particulièrement mouvementées par rapport aux autres années. Le nombre élevé d'infractions à la loi enregistré en 1997, par exemple, est imputable, selon les services de la police, au repli sur Niamey des délinquants de Côte-d'Ivoire sévèrement réprimés par la police de ce pays et du Burkina Faso. L'observation des principes du droit par la police de Niamey, sous la surveillance des associations des droits de l'homme avait fait de la capitale du Niger un refuge des bandits traqués ailleurs. Cambriolages, agressions et vols de tous genres y furent donc nombreux (Fig. 2).
L'absence de séries longues ne nous permet pas de démontrer une augmentation de la criminalité liée ni à l'urbanisation galopante ni à l'instauration d'un Etat de droit et encore moins à la crise économique. Les vicissitudes de la vie politique, notamment le passage entre Etat de droit et Etat d'exception qui a caractérisé la période, ne transparaissent pas non plus dans l'analyse des données. La répartition par saison des délits de l'année 2000, par contre, montre une forte corrélation, qui demande cependant à être vérifiée sur une longue période, entre la criminalité et la présence des ruraux en ville corroborant ainsi les études de D. Porrou (1981). La figure ci-après montre en effet une augmentation de la criminalité d'octobre/novembre, période correspondant aux faibles récoltes de mars/ mai suscitées par la période sèche et chaude qui a incité les jeunes ruraux à déserter les campagnes, non seulement pour chercher un travail mais aussi pour permettre à ceux qui restent de pouvoir manger à leur faim jusqu'à la prochaine récolte (Fig. 3).

Au niveau du TPIN, les données collectées entre 1980 et 1998 ne concernent que les vols dont le nombre a aussi évolué. On peut cependant identifier 4 périodes (Fig. 4):

- De 1980 à 1985, la croissance est régulière: de 303 on est passé à 855 cas. Cela paraît paradoxal car nous sommes ici en plein régime d'exception disposant d'une police répressive qui s'embarrasse peu du respect des droits de l'homme.

- Un redressement de la situation est peut-être intervenu en 1986/87 si l'on se fie à la baisse de la crimi- 


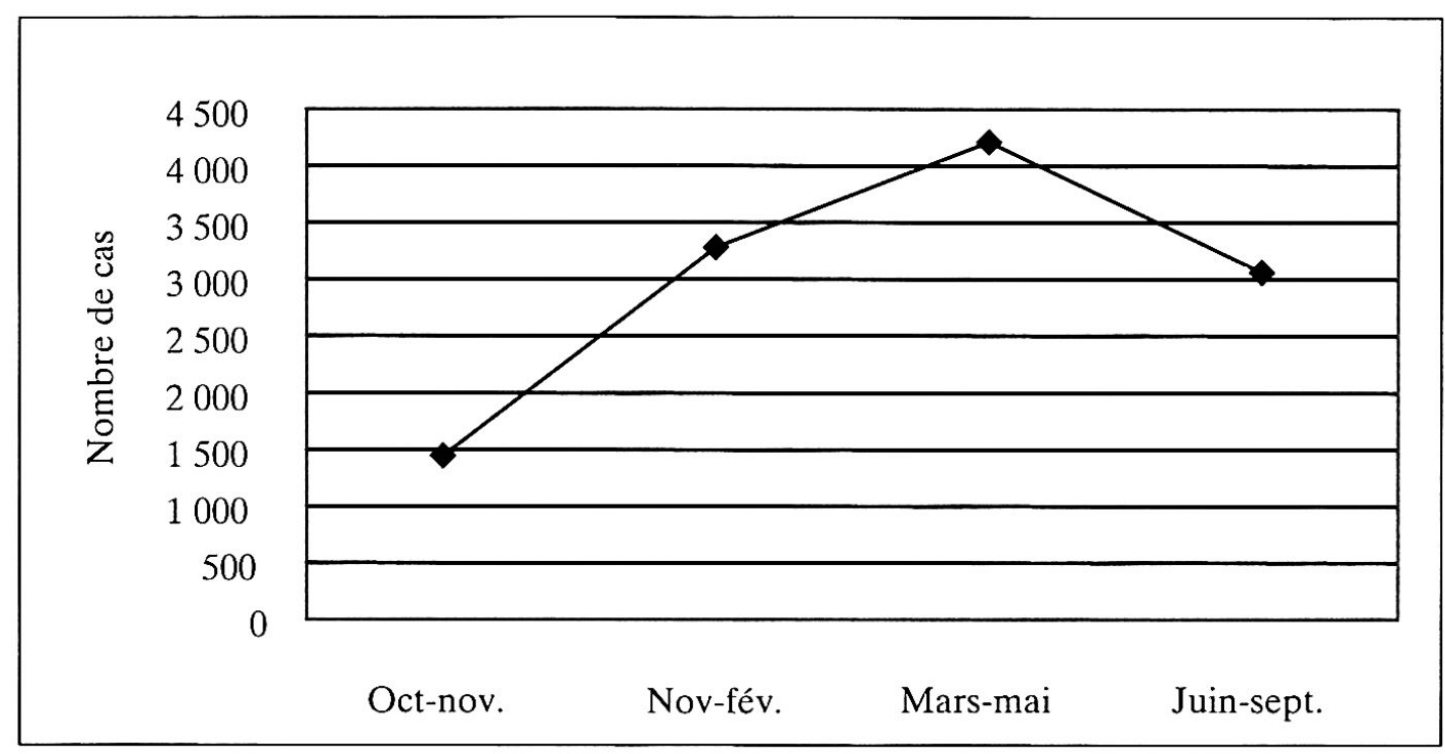

Fig.3: Evolution de la criminalité selon les saisons

Development of criminality according to seasons

Entwicklung der Kriminalität - nach Jahreszeit

Source: Direction générale de la police nationale, Service central de l'identité judiciaire, rapports d'activités, 1991 à 2000

nalité qui reprend de plus belle entre 1988 et 1992 avec le changement d'homme à la tête de l'Etat. C'est la période dite de la décrispation politique.

- De 1993 à 1995, la criminalité s'accroît de nouveau après une courte pause en 1993. C'est la période de la transition démocratique et des élections présidentielles et législatives avec ses incertitudes politiques et ses crises économiques.

- De 1996 à 1998, la criminalité baisse de nouveau, bien que sur le plan politique la période soit trouble: le Niger a en effet enregistré deux coups d'Etat et les fonctionnaires plusieurs mois d'arriérés de salaire.

L'analyse des statistiques du TPIN révèle l'existence de liens entre les vols et les crises que le Niger a traversés au cours de la période 1991/99. N'oublions pas que le pays fut aussi durement frappé par plusieurs sécheresses qui ont poussé davantage de ruraux à venir gonfler le nombre de mendiants de Niamey.

\subsection{Nature des principaux délits commis}

Expurgés des rafles, les crimes et délits commis, recensés par le SCIJ, sont par ordre décroissant les vols ( $42 \%$ des infractions à la loi), les coups et blessures volontaires $(17 \%)$, les accidents $(10 \%)$, les divers abus de confiance $(9 \%)$, les escroqueries $(9 \%)$ et les troubles de l'ordre public (3\%). Ensemble, ces infractions représentent quatre délits sur cinq. Les cinq infractions à la loi les plus importantes, venant après ce premier groupe (menace de mort, troubles de l'ordre public, consommation de stupéfiants, recels, et ivresse "publique et manifeste» ne représentent qu'une infraction sur 10. Le reste des atteintes à la loi, au nombre de 18 , ne représente que $7 \%$ des infractions.

Les jeux de hasard, la prostitution, le vagabondage et la mendicité qui sont des délits punis par la loi sont très fréquents. Ils sont, cependant, sanctionnés avec peu de zèle par les Services de Police. La mendicité qui est devenue un phénomène de société est même tolérée malgré la gêne qu'elle constitue. Les meurtres souvent évoqués par les organes de presse représentent moins de $1 \%$ des infractions à la loi à Niamey.

La distinction des infractions en délits et en crimes montre la nette prédominance du premier qui représente plus de 9 cas sur 10. Ici aussi ce sont les vols simples (à la tire et à l'étalage) qui prédominent. Les vols qualifiés (en réunion, avec effraction ou suivi de mort) sont assez rares. Si les vols sont particulièrement nombreux, c'est parce que les receleurs le sont aussi: ils représentent $2 \%$ des délinquants interpellés par la police. Après les vols simples, ce sont les coups et blessures qui viennent en seconde position. La promiscuité dans laquelle vit une bonne partie de la population engendre souvent des bagarres sans gravité: l'habitat de type cour regroupant plusieurs ménages est un facteur favorisant les altercations entre enfants et entre parents. Ici aussi, les délits sans gravité sont particulièrement nombreux à l'instar des vols simples. 


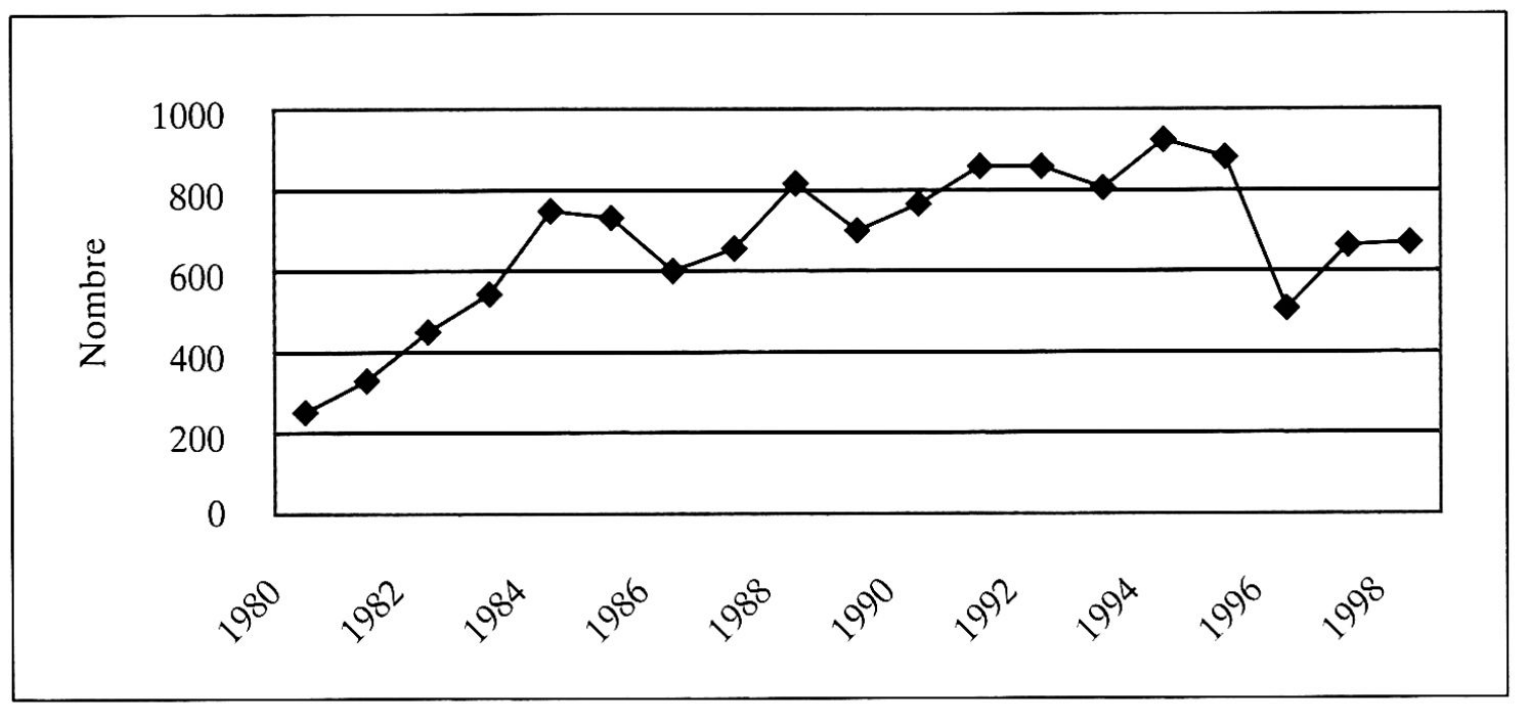

Fig. 4: Evolution du nombre de voleurs enregistrés par le tribunal de 1ère instance de Niamey Development of number of thieves registered at the trial court in Niamey

Zahl der gerichtlich erfassten Diebe in Niamey

Source: Direction générale de la police nationale, Service central de l'identité judiciaire, rapports d'activités, 1991 à 2000

Par ailleurs, il est aussi important d'évoquer trois phénomènes liés à l'urbanisation, à savoir les accidents de circulation ( $95 \%$ des cas), puis les escroqueries, enfin, les autres abus de confiance fréquents dans une ville de la taille de Niamey où l'anonymat permet aux délinquants d'opérer avec une facilité relative.

\subsection{Le profil social des délinquants}

Qui sont les auteurs des crimes et délits? Les données fournies par le TPIN indiquent que les délinquants sont, de préférence, recrutés surtout parmi les jeunes $(61 \%)$ et les groupes de travailleurs à bas salaire. Nous constatons que la délinquance juvénile occupe une place importante et que le profil social du délinquant ne diffère pas de celui de la population pauvre.

En observant des prisonniers localisés dans le système pénitentiaire de Niamey, TsaHIROU (2000) conclut que les délinquants se recrutent surtout au sein de la population de travailleurs indépendant à bas revenu $(60 \%$ des détenus pour cause de vols) et dans une moindre mesure parmi les sans emploi (30\%). L'étude montre donc que les deux tiers des personnes condamnées pour vol avaient un emploi au moment de leur arrestation. Si l'étude démystifie l'image que le délinquant n'a pas d'emploi, elle montre cependant que deux voleurs sur cinq sont analphabètes et un quart a fréquenté l'école coranique. Ainsi les délinquants appartiendraient à la catégorie des petites gens sans instruction, exerçant surtout un petit métier dans le secteur informel. Ces petites gens appartiennent à la classe des pauvres qui représentaient en 1993208000 habitants (Ministère du Plan et de la Planification RégioNALE 1989). Le dénuement serait, d'ailleurs, selon les travaux de Tsahirou, le premier mobile invoqué par les voleurs. La répartition des voleurs selon leur profession avant leur incarcération montre en effet que plus le niveau de formation est élevé, plus l'occurrence de crimes diminue: ainsi les diplômés ne représentent que $1 \%$ des voleurs, les autres salariés $12 \%$; ensemble ils ne totalisent que $13 \%$ des voleurs contre $35 \%$ pour les non salariés et $52 \%$ pour les chômeurs.

L'image du voleur en tant qu'individu pauvre, et confirmée par l'analyse de la typologie des crimes, montre que la plupart des délinquants sont des délinquants accidentels ou occasionnels: les données fournies par le TPIN révèlent en effet que les vols individuels et primaires représentent respectivement $88 \%$ et $86 \%$ des cas de vols.

\subsection{Mobilisation des Niaméens contre la criminalité}

Les multiples vols dont sont victimes les Niaméens les ont poussés à se mobiliser pour lutter contre ce fléau. C'est ainsi que la quasi totalité des quartiers périphériques dispose de Yam banga dont la fréquence des patrouilles est intimement liée à la recrudescence des vols enregistrés dans les quartiers mais aussi à l'acceptation des résidents à débourser la somme de $500 \mathrm{~F}$ CFA par concession. Il convient à ce niveau de mentionner que bien que bénéficiant d'une autorisation 
d'exercer de la police, celle-ci ignore leur nombre et leur localisation. Ceci n'est pas sans danger pour les citoyens car les Yam banga peuvent très bien verser dans la délinquance.

La seconde stratégie de lutte contre les vols est la protection adéquate des appartements au stade de la conception des bâtiments, par l'adoption de cadres de portes et fenêtres et de verrous appropriés, c'està-dire difficiles à forcer pour les cambrioleurs, par le rehaussement des murs de clôtures surmontés de pointes ou de tessons de bouteilles, et, enfin, par la plantation des bougainvilliers tout le long de la clôture.

La troisième stratégie est le recours aux sociétés de gardiennage. Très peu de familles peuvent s'offrir les services de l'une des deux sociétés de gardiennage de la place. Les coûts sont hors de portée des bourses du citoyen moyen. Il en est de même du recours à la souscription à une assurance contre le cambriolage à laquelle seules les sociétés disposant d'énormes moyens peuvent avoir recours.

Enfin, de plus en plus de Niaméens évitent de faire l'étalage de leur richesse: les bijoux sont de moins en moins portés avec extravagance par les femmes. Les citoyens qui font des retraits importants pour leur propre compte à la banque font attention à ne pas être suivis par les délinquants, de peur d'être inquiétés une fois la nuit tombée.

\section{Conclusion}

Les données fournies aussi bien par le Tribunal de première instance de Niamey que par le Service central de l'identité judiciaire ne nous permettent pas, malgré les présomptions, d'affirmer avec force que l'augmentation de la criminalité dans la communauté urbaine de Niamey est la résultante de la croissance rapide de la ville. Les moyens dérisoires dont dispose la police et l'extension spatiale de la ville ont engendré à tort ou à raison des réflexes sécuritaires chez les Niaméens. Mal encadrés, ces réflexes peuvent à leur tour générer des problèmes de sécurité. Ainsi, en l'absence de normes quant aux comportements à adopter pour dissuader les voleurs, les Niaméens transforment leur habitation en bunker sans envisager comment échapper à un incendie provenant de l'intérieur de l'habitation. De même, les comités de défense des quartiers, les Yam banga, peuvent, sous l'effet conjugué de la misère et du refus des ménages de «s'acquitter de la redevance mensuelle» de 500 F CFA, soit se transformer en délinquant, soit être de connivence avec les voleurs. L'Etat se doit de se donner les moyens adéquats pour garantir, à chaque citoyen de la ville de Niamey ou d'ailleurs, la sécurité dont il a besoin pour son épanouissement. Cela passe aussi par un accès de tous les Nigériens à la formation et à l'emploi. Au delà de ce vœux pieux, l'Etat peut cependant canaliser les initiatives des populations, afin de prévenir les infractions à la loi.

\section{Bibliographie}

Direction générale de la police nationale, Service CENTRAL DE L'IDENTITÉ JUdiCIAIRE (1991 à 2000): Rapports d'activités. - Niamey.

GrÉGOIRE, E. (1992): L'encadrement et l'insertion des jeunes par les filières marchandes et religieuses dans une ville sahélienne (Maradi, Niger). - In: Jeunes, ville, emploi: Quel avenir pour la jeunesse africaine? Acte du colloque, Paris, 26-29 octobre 1992: 168-172.

Ministère des Finances et du Plan, Secrétariat Général, Direction de la Statistique et des Comptes Nationaux (SG/DSCN): Enquête sur le budget et la consommation des ménages au Niger: 1989/19901992/1993, Profil de la pauvreté. - Niamey.

Ministère du Plan, Secrétariat Général, Direction de la Statistique et des Comptes Nationaux (1989): Enquête nationale sur le secteur informel et la petite entreprise. Diagnostic du secteur informel urbain au Niger. Importance du secteur, problèmes des entrepreneurs, fiscalité, investissement et dynamique du secteur. - Niamey.

Ministère du Plan et de la Planification Régionale, Bureau Central de Recensement (1989): Deuxième recensement général de la population, 1988, résultats non-publiés. - Niamey.

Motcho, K.H. (1991): Cadre de vie systèmes de santé à Niamey (Niger). - Bordeaux: Université Michel de Montaigne de Bordeaux III, UFR de Géographie et de gestion des espaces.

Motcho, K.H. \& M. Ben AdJ (1998): La lutte contre la pauvreté au Niger: solutions actuelles et perspectives. In: Actes du colloque du Département de géographie/ FLSH/UAM: Urbanisation et pauvreté en Afrique de l'Ouest, Niamey, 4-6 juillet 1996, Annales de l'Université Abdou Moumouni de Niamey, $\mathrm{N}^{\circ}$ hors série: 257-272.

Portou, D. (1981): Délinquance juvénile et urbanisation au Niger et au Nigéria. - In: Cahiers Etudes africaines 81-82, XXI-1-3: 111-127.

Souley, B. (1995): L'enseignement coranique dans la Communauté Urbaine de Niamey: Organisation fonctionnement - population. - Texte inédit de l'Office de recherches scientifiques et travaux d'outre-mer (ORSTOM), Niamey.

TSahirou, M. (2000): La délinquance en milieu urbain au Niger: évolution des vols dans la communauté urbaine de Niamey.- Mémoire de Maîtrise, Sociologie, Université du Bénin, Lomé. 


\section{Résumé: Croissance urbaine et insécurité dans la ville de Niamey}

Née de la colonisation, la ville de Niamey comptait 1730 habitants en 1931 et plus de 15000 en 1953, soit une croissance annuelle de $7 \%$. A partir de 1970, ce taux augmente pour atteindre $10 \%$. En 1988 , la capitale du Niger comptait 400000 habitants. Aujourd'hui, elle en comporte près de 700000 . L'extension de la ville a été tout aussi rapide: en 1970, Niamey couvrait 1367 ha, alors que sa superficie atteint plus de 10000 ha aujourd'hui. Cette urbanisation galopante pose avec acuité des problèmes de sécurité. Vols à la tire, cambriolages et braquages, contre lesquels les quelques fonctionnaires de la police sont impuissants, font aujourd'hui partie du vécu des Niaméens.

Cette insécurité grandissante est malheureusement le résultat de deux facteurs principaux qui sont intimement liés: la paupérisation grandissante de la population et la faiblesse du mode d'encadrement des jeunes. La délinquance juvénile prend de plus en plus d'ampleur, comme en témoigne le très grand nombre d'enfants de la rue qui, à l'occasion, commettent de petits larcins ou, au pire, sont les indicateurs des grands délinquants.

\section{Summary: Urban Growth and Insecurity in Niamey}

Founded during colonial times, the city of Niamey had 1730 inhabitants in 1931. Twenty-two years later, the population had increased to 15710 , representing an annual growth of $7 \%$. After 1970, this growth increased to $10 \%$. By 1988 , the capital had 400000 inhabitants. Currently, 700000 people live in the city. The expansion of the city reflects this rapid demographic growth: in 1970, Niamey covered 1367 ha, today the city spreads over $10000 \mathrm{ha}$. This rapid growth was and still is associated with various difficulties in the areas of accommodation, health and especially security.

This increasing insecurity is the result of two main factors which are closely linked: increasing poverty plus lack of education and support of the younger generation. Juvenile delinquency is becoming more and more prevalent as indicated by the increasing number of street children who commit petty thefts when the occasion presents itself or at worst could become involved in organised crime.

\section{Zusammenfassung: Urbanes Wachstum und Unsicherheit in Niamey}

Gegründet in der Kolonialzeit zählte Niamey 1931 1730 Einwohner. Zwanzig Jahre später war die Bevölkerungszahl auf 15710 gestiegen, was einem jährlichen Zuwachs von 7\% entspricht. Nach 1970 stieg die Wachstumsrate auf 10\%, 1988 zählte die Hauptstadt 400000 Einwohner. Zur Zeit sind es 700000 Einwohner. Die Ausdehnung der Stadt widerspiegelt diesen rapiden Bevölkerungszuwachs: 1970 erstreckte sich Niamey auf 1367 ha, gegenwärtig auf 10000 ha. Dieses schnelle Wachstum führte zu verschiedenen Problemen beim Wohnen, bei der Gesundheit und vor allem der Sicherheit.

Die zunehmende Unsicherheit ist das Resultat von zwei Hauptfaktoren, die eng miteinander verbunden sind: die zunehmende Armut und das Fehlen von Erziehung (Ausbildung) und Unterstützung der jüngeren Generation. Die Jugendkriminalität steigt stark an, was sich an den zahlreichen Kindern auf der Strasse zeigt, welche entweder kleinere Diebstähle begehen oder schlimmer, die Indikatoren des organisierten Verbrechens sind.

\section{Orientations didactiques}

- Quelles sont les causes possibles des problèmes sociaux croissants?

- Les conditions de l'urbanisation et la typologie de l'habitat sont-elles des facteurs à prendre en considération pour aborder le problème de la sécurité dans les villes africaines?

- De quelle façon la ville se structure-t-elle et quelle est la forme de violence qui s'exprime dans ses diverses parties?

- Quelle est l'influence exercée sur la sécurité par le faible âge moyen et la forte croissance démographique?

- Quelles sont les formes de délits commis et quels en sont les auteurs?

- En examinant la ville de Niamey, en pleine expansion, nous constatons qu'elle comptait en 1960 seulement 30000 hab. En 2002, la population a été évaluée à près de 700000 hab., en 2020, elle comptera 1700000 hab. dispersés sur un vaste territoire constitué de lotissements produits par la municipalité et surtout de lotissements sauvages. Comment pourrat-on assurer la sécurité publique et par qui?

- Comment combat-on la criminalité à Niamey?

Dr. Kokou Henri Motcho, Maître-Assistant de Géographie, Faculté des Lettres et Sciences Humaines, Université Abdou Moumouni, BP 418 Niamey, Niger. e-mail:depgeo@intnet.ne

\section{Manuskripteingang/received/manuscrit entré le 17.2.2004}

Annahme zum Druck/accepted for publication/accepté pour l'impression: 20.8.2004 